\title{
Keine ZNS-Nebenwirkungen bei der OAB-Therapie mit Trospiumchlorid
}

_ In der Therapie der überaktiven Blase (OAB) hat der Wirkstoff Trospiumchlorid, (Spasmex ${ }^{\circledast}$ ), einen entscheidenden Vorteil gegenüber anderen Wirkstoffen: Er dringt nicht in das Gehirn ein und verursacht deshalb keine ZNS-Nebenwirkungen wie beispielsweise Sedation, Erschöpfung, Halluzinationen oder Gedächtnisstörungen.

Wie Prof. Dr. Joachim Geyer vom Institut für Pharmakologie und Toxikologie der JustusLiebig-Universität Gießen erläutert, liegt der Vorteil von Trospiumchlorid nicht nur an seiner positiven Ladung und hohen Polarität, die ein Eindringen in das Gehirn und damit zentrale Nebenwirkungen unterbinden. Untersuchungen an Mäusen zeigten, dass auch der MDR1-Effluxtransporter, auch PGlycoprotein (P-gp1)-Transporter genannt, die Gehirngängigkeit von Trospiumchlorid limitiert. Damit konnte Geyer belegen, dass der MDR1-Effluxtransporter an einer intak- ten Blut-Hirn-Schranke eine zusätzliche, biologische Barriere für Trospiumchlorid zum Gehirn ist.

Ebenso konnte Geyer erst kürzlich am Mausmodell zeigen, dass der Wirkstoff Trospiumchlorid auch für ältere Patienten sehr gut geeignet ist. Im Tierversuch nahm die Gehirngängigkeit von Trospiumchlorid bei älteren Mäusen nicht zu.

"Auch bei älteren OAB-Patienten muss unter Trospiumchlorid nicht mit einem Auftreten relevanter ZNS-Nebenwirkungen gerechnet werden", so Geyer. Somit ist gerade für ältere Patienten Trospiumchlorid die ideale Therapieoption bei $O A B$, da es nicht wie andere Medikamente die im Alter gehäuft auftretenden Schlaf-, Lern- und Gedächtnisstörungen hervorruft.

Nach Informationen von

Dr. R. Pfleger, Bamberg
uriVesc ${ }^{\circledast}$ jetzt günstiger

Der Preis für die TrospiumchloridRetardkapsel uriVesc ${ }^{\circledast}$ ist seit kurzem niedriger, denn das Anticholinergikum ist seit dem 15. Januar festbetragsgeregelt. Die 28er-Packung kostet jetzt 29 EUR, die 84er-Packung 62,82 EUR. Durch diese neue Preisgestaltung weist das Medikament die niedrigsten Tagestherapiekosten unter allen gängigen Blasenspasmolytika auf.

Die Einmalgabe des Trospiumchlorids zeichnet sich durch eine gute Verträglichkeit und hohe Wirksamkeit aus. Die Galenik des Medikaments führt zu einer sofortigen Freisetzung im Dünndarm und einer ph-abhängigen verzögerten Freisetzung im Dickdarm. Die Therapietreue ist dabei dank des guten Nebenwirkungsprofils hoch: Lediglich 10,7\% der Patienten unter uriVesc ${ }^{\circledR}$ gaben als häufigste unter Blasenspasmolytika beobachtete Nebenwirkung Mundtrockenheit an. Metabolische Interaktionen mit anderen Arzneimitteln gibt es ebenfalls nicht.

Nach Informationen von Rottapharm|Madaus, Köln

Alterskrankheiten des Mannes bei Hämophiliepatienten

Hämophiliepatienten haben heute eine nahezu normale Lebenserwartung. Daher haben die meist männlichen Patienten nun auch mit den für ihr Geschlecht typischen Alterskrankheiten zu kämpfen. Hormonumstellungen, Metabolisches Syndrom, Osteoporose und hepatozelluläres Karzinom gewinnen in dieser Patientengruppe zunehmend an Bedeutung. Die Substitutionstherapie mit einem Faktor-VIII-Präparat wie beispielsweise Kogenate ${ }^{\circledR}$ BAYER bleibt dabei in vielen Behandlungskonzepten unverzichtbar. Insgesamt ist der ältere hämophile Mann damit eine therapeutische Herausforderung, die es im interdisziplinären Netzwerk zu meistern gilt. So spielt zum Beispiel der sinkende Testosteronspiegel eine Rolle bei der Zunahme des Bauchumfangs. Diese hat nicht nur unschöne ästhetische, sondern auch negative gesundheitliche Folgen, betonte Androloge Prof. Michael Zitzmann.

Nach Informationen von

Bayer Vital, Leverkusen
Nach Informationen von

Abbott Arzneimittel, Hannover höhere Abheilungsraten bachtet als bei Männern (68\% versus 53,7\%).
Die anatomischen Verhältnisse der Frau ich sein. Darüber hinaus waren die Rezidivextrakt niedrig: Sie lagen zwischen $4,1 \%$ und ups nach kompletter Abheilung.

Um den optimalen Therapieerfolg zu erzielen scheidend, daher sollten Betroffene darüber dreimal täglich mit dem Finger dünn auf Die Therapie solte bis zum vollstandigen Abheilen der Warzen durchgeführt werden. Die maximale Anwendungsdauer beträgt 16 vor allem zwischen der zweiten und vierten Behandlungswoche zu entzündlichen Lokalreaktionen kommen, welche die Patienten in der Regel gut tolerieren. 